Rev. Elev. Méd. vét. Poys trop., 1968, 21, 4 (479-491).

\title{
Stérilisation des mâles de Glossina tachinoides West. par irradiation aux rayons gamma
}

\author{
por J. ITARD
}

\begin{abstract}
RÉSUMÉ
Dans la perspective d'une application pratique de la méthode de lutte par lâchers de mâles slériles, les conséquences de l'ırradiation gamma de môles adultes de Glossino tachinordes West. ont été étudiées.

Les doses d'irradiation optimales se situent entre 15.500 ef 17.000 rads. A ces doses, la stérılité des mâles esł totale, mais leur vigueur sexuelle n'est pas diminuée et leur longévité demeure satısfaisante.

Ces doses sont inférieures à celles qui sont nécessaires pour obtenır une stérilıté tctale chez les mâles de Glossina morsitans marsitans Newst.
\end{abstract}

\section{1. - INTRODUCTION.}

Les premières recherches sur les possibilités de stérilisation des glossınes par irradiation gamma ont été réalisées par POTTS, en 1958, sur des pupes de Glossina morsitans importées d'Afrique Orientale. Bien que, comme le souligne l'auteur, les résultats n'aient guère été probants, en raison du petit nombre d'expérimentations, POTTS conclut que les tsé-tsés peuvent être stérilisées à des doses comprises entre 6.000 ef 12.000 r. e. p. et que les mouches irradiées sont compétitives avec les mouches normales, bien que leur longévité soit réduite.

Ces recherches ont été reprises, à partir de l'année 1964, en Rhodésie par DEAN. DAME, FORD et PHELPS, sur des pupes et des adultes de Gl. morsitans orientalıs et Gl. pollidipes et ont porté à la fois sur l'utilisation des produits chimiostérilisants et sur l'action des rayonnements gammo.

Des essaıs réalısés en Rhodésie, it ressort que l'irradiation gamma, à des doses comprises entre 8.000 et 15.000 rads, de pupes, d'âge inconnu, récoltées dans la nature, diminue de 95 p. 100 le pouvoir reproducteur des mouches mâles qui éclosent dans la semaine suivant le traitement. Les mâles qui éclosent pendant la $2^{\mathrm{e}}$ et la $3^{\mathrm{e}}$ semaine suivant le traitement sont complètement stériles d̀ 9.000 et 4.000 rads, respectivement. Les femelles sont complètement stérilisées à 1.000 rads. La survie des pupes irradiées et des adultes qui en éclosent est réduite à la fois par l'accroissement des doses d'irradiation et par la diminution de l'âge des pupes au moment de l'irradiation. Les mouches mâles. sont plus sensibles aux rayons gamma que les femelles.

Les mâles adultes irradiés entre 8.000 et 16.000 rads peu après l'éclosion ont également une fertilité rédulte de $95 \mathrm{p}$. 100 , mas is vivent moins longtemps que les mâles non irradiés. La stérilité est restée permanente pendant les 45 jours d'expérımentation. Le sperme des mâles irradiés est mobıle, et apparemment normal. Les mâles irradiés sont sexuellement aussi compétıtıfs que les mâles normaux.

Nous avons à la fin de l'année 1966, dans le but de nous familiariser avec cette méthode. réalisé des expériences semblables sur des lots de pupes d'âge divers mais connu et des mâles adultes âgés de $24 \mathrm{~h}$, de Gl. morsitans morsitons 
élevés au laboratoire d'Entomologie de l'I. E. $M . V$. T., qui ont été irradiés au moyen d'un irradiateur au Césium 137. Les résultats obtenus avec les pupes ont confirmé ceux qui ont été publiés par les chercheurs rhodésiens. La dose d'irradiation optimum semble se situer autour de 7.000 rads et doit être appliquée à des pupes âgées de plus de 12 jours.

Cependant de meilleurs résultats furent obtenus, tant en ce qui concerne la longévité que la diminution de fertilité, par irradiation des mâles adultes. La stérilité totale a été obtenue à des doses comprises entre 20.000 rads et 25.000 rads, tout en permettant une longévité 50 p. $100\left(^{*}\right)$ de 28 à 24 jours.

II nous a paru alors intéressant de reprendre ces études de façon plus complète, en irradıant des mâles adultes de Glossina tachinoides. Cette espèce, dont l'aire de répartition s'étire en latttude entre $4^{\circ}$ nord ef $14^{\circ}$ nord, et en longitude depuis $10^{\circ}$ ovest jusqu'd $21^{\circ}$ est, avec les îlots en République du Soudan et en Ethiopie, est un important vecteur des trypanosomiases humaines et animales. Plus petite que Gl. morsitons, elle a besoin d'une humidité plus importante et supporte difficilement la sécheresse. Elle paraît donc plus fragile ef pourrait être plus sensible aux effets des rayonnements gamma que $G$. morsitans.

Dans la perspective d'une application pratique de la méthode de lutte contre Gl. tachinoides par lâchers de mâles stériles, il était donc nécessaire de préciser les doses d'irradiation à appliquer à cette espèce.

\section{II. - MATÉRIEL ET TECHNIQUES}

Les insectes utilisés proviennent d'un élevage permanent réalisé au laboratoire d'Entomologie de l'I. E. M.V. T. depuis la fin de l'année 1966.

Les mouches sont nourries sur oreilles de lapin; les femelles sont accouplées d̀ l'âge de 3 jours avec des mâles âgés de 7 jours; au $60^{\mathrm{e}}$ jour suivant l'éclosion, la production de pupes pour, 100 femelles accouplées, est au minimum de 282 pupes.

(*) Par analogie avec la période des corps radioactifs, que l'on peut définir comme le temps au bout duquel la moitié du nombre initial d'atomes a disparu, nous entendons par longévité 50 p. 100 le temps au bout duquel it reste la moitıé du nombre initial d'indıvidus vivants.
Les irradiations ont été effectuées au moyen: d'un irradiateur au Cobalt 60 d'une puissance de 35.056 rads-heure mis à notre disposition par le Service de Biophysique du Centre d'Etudes. Nucléaires de Saclay.

Nous avons constitué 13 lots de 40 mâles et un lot de 38 mâles, dont l'âge, au moment de l'irradiation, était compris en 1 et 9 jours. Les proportions des mâles aux différents âges. étaient pratiquement identiques dans chaque lot (Tableau 1).

Les insectes placés dans des cages de type Roubaud, ont été transportés dans des boîtes isothermes jusqu'au Centre d'Etudes Nucléaires.

Les irradiations ont été effectuées à deux époques différentes; ,une premıère séance eut lieu le 6 décembre 1967 et comprenait :

un lot de 40 mâles témoins, un lot de 40 mâles irradiés à 2.000 rads, un lot de 40 mâles irradiés à 6.000 rads, un lot de 40 mâles irradiés à 10.000 rads, un lot de 40 mâles ırradiés à 15.000 rads, un lot de 40 mâles irradiés à 20.000 rads, un lot de 40 mâles irradiés à 25.000 rads.

La deuxième séance fut réalisée le 5 mars 1968: et comprencit :

un lot de 40 mâles témoins,

deux lots de 40 mâles irradiés à 15.500 rads, un lot de 40 mâles irradiés à 17.000 rads, un lot de 38 mâles irradiés à 17,000 rads, deux lots de 40 mâles irradiés à 18.500 rads.

Les durées d'exposition ont été comprises entre $3 \mathrm{mn} 26 \mathrm{~s}$ pour 2.000 rads ef $42 \mathrm{mn} 55 \mathrm{~s}$ pour 25.000 rads.

\section{III. - RÉSULTATS}

\section{1. - Longévité des mâles témoins et des mâles irradiés (graphique 1,2 ef 3 ).}

L'âge moyen des mâles témoins et des mâles Irradiés, au moment de l'irradiation, est de 4 jours pour les lots du 6 décembre 1967 ef de 6 jours pour les lots du 5 mars 1968 (Tableau 1).

Les mâles témoins ont une longévité 50 p. 100 de 78 jours pour le lot du 6 décembre et de 77 jours pour le lot du 5 mars. La longévité maximum dépasse 93 jours (les survivants, soił 30 p. 100, ont été sacrifiés le 93 jour). 
Retour au menu

TABLEAU No 1. - Age des mâles au moment de l'irradiation.

\begin{tabular}{|c|c|c|c|c|c|c|c|c|}
\hline \multirow{2}{*}{$\begin{array}{c}\text { Dateo } \\
\text { d'éclosion }\end{array}$} & \multicolumn{7}{|c|}{ Nombre de mâles (lots du 6.12.67) } & \multirow{2}{*}{$\begin{array}{c}\text { Age } \\
\text { en } \\
\text { jours }\end{array}$} \\
\hline & Têmoins & $2.000 \mathrm{rads}$ & $6.000 \mathrm{rads}$ & 10.000 rads & 15.000 rads & $20.000 \mathrm{rads}$ & $25.000 \mathrm{rads}$ & \\
\hline 29.11 .67 & 4 & 4 & 4 & 4 & 4 & 4 & 4 & 8 \\
\hline 30.11 .67 & 5 & 5 & 5 & 5 & 5 & 5 & 5 & 7 \\
\hline 1.12 .67 & 3 & 3 & 3 & 3 & 3 & 3 & 3 & 6 \\
\hline 2.12 .67 & 4 & 4 & 4 & 4 & 4 & 4 & 4 & 5 \\
\hline 3.12 .67 & 9 & 9 & 9 & 9 & 10 & 10 & 9 & 4 \\
\hline 4.12 .67 & 2 & 3 & 3 & 3 & 2 & 2 & 2 & 3 \\
\hline 5.12 .67 & 6 & 5 & 5 & 5 & 5 & 5 & 5 & 2 \\
\hline 6.12 .67 & 7 & 7 & 7 & 7 & 7 & 7 & 8 & I \\
\hline $\begin{array}{l}\text { Age moyen } \\
\text { (en jours) }\end{array}$ & $4, .15$ & 4,17 & 4,17 & 4,17 & 4,20 & 4,20 & 4,12 & \\
\hline Dates & \multicolumn{7}{|c|}{ Nombre de mâles (lots du 5.3 .68 ) } & Age \\
\hline$d^{\text {téclosion }}$ & Têmolns & \multicolumn{2}{|c|}{$15.500 \mathrm{rads}$} & \multicolumn{2}{|c|}{17,000 rads } & \multicolumn{2}{|c|}{$18.500 \mathrm{rads}$} & jours \\
\hline 26.2 .68 & 6 & 6 & 6 & 6 & 6 & 6 & 6 & 9 \\
\hline 27.2 .68 & 6 & 6 & 6 & 6 & 5 & 6 & 6 & 8 \\
\hline 28.2 .68 & 5 & $s$ & 5 & 5 & 5 & 5 & 5 & 7 \\
\hline 29.2 .68 & 6 & 6 & 6 & 6 & 5 & 6 & 6 & 6 \\
\hline 2.3 .68 & 5 & s & 5 & 5 & s & 5 & 5 & 5 \\
\hline 2.3 .68 & 5 & $\mathbf{s}$ & 5 & 5 & 5 & 5 & 5 & 4 \\
\hline 4.3 .68 & 7 & 7 & 7 & 7 & 7 & 7 & 7 & 2 \\
\hline $\begin{array}{l}\text { Age moyen } \\
\text { (en jours) }\end{array}$ & 5,8 & \multicolumn{2}{|c|}{5,8} & 5,8 & 5,7 & \multicolumn{2}{|c|}{5,8} & \\
\hline
\end{tabular}

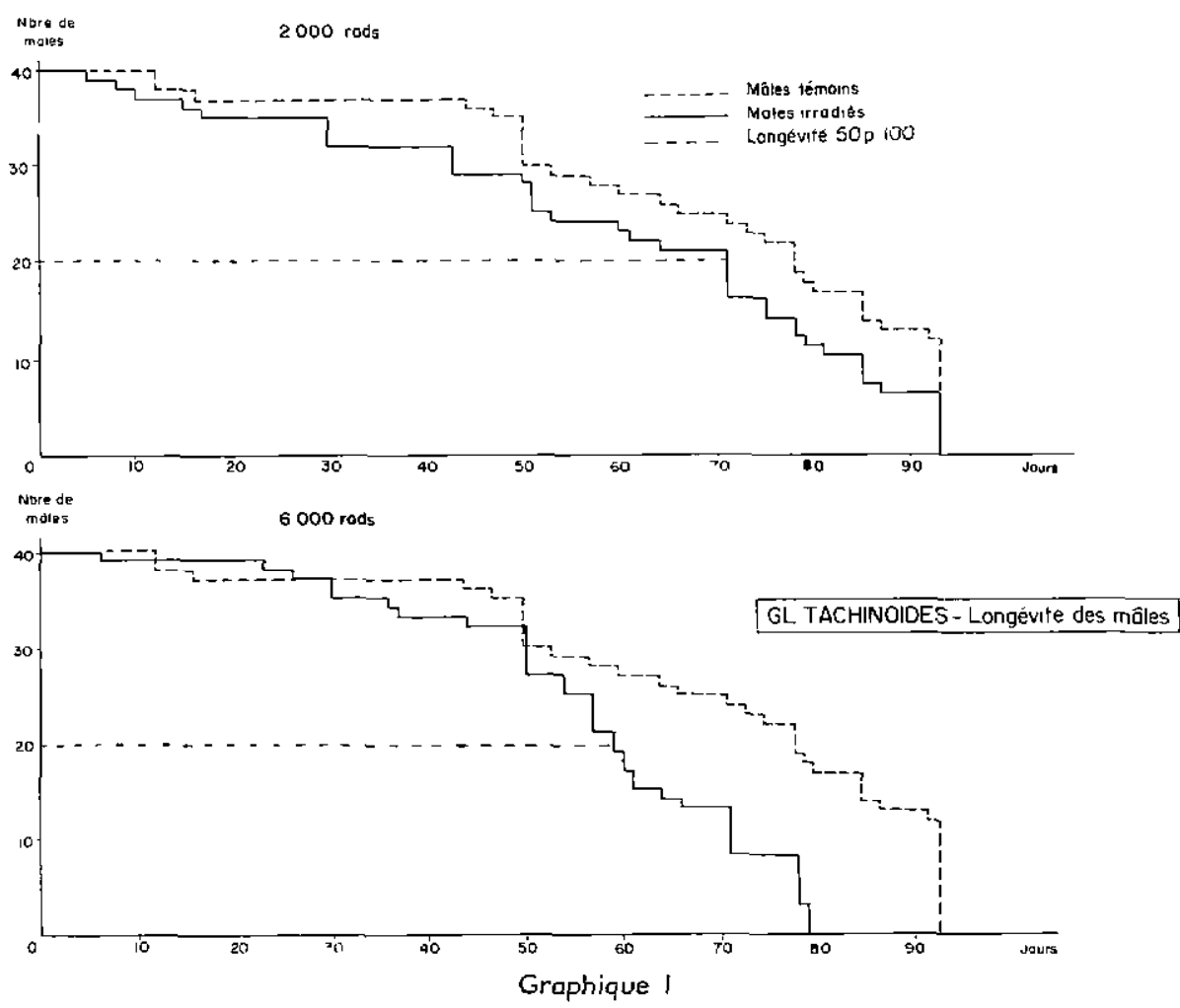




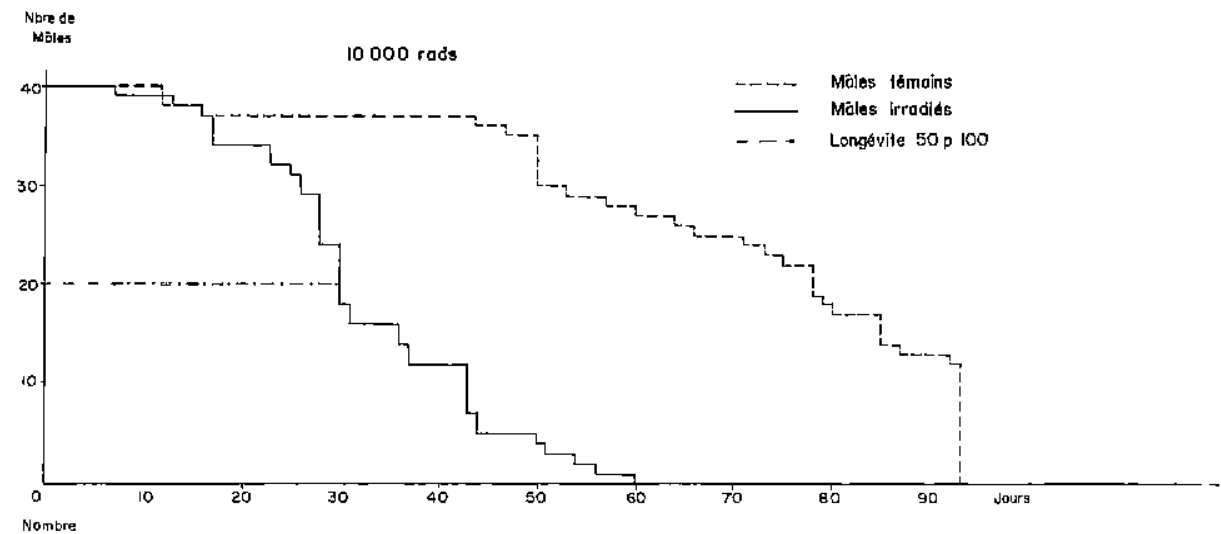

Nambre
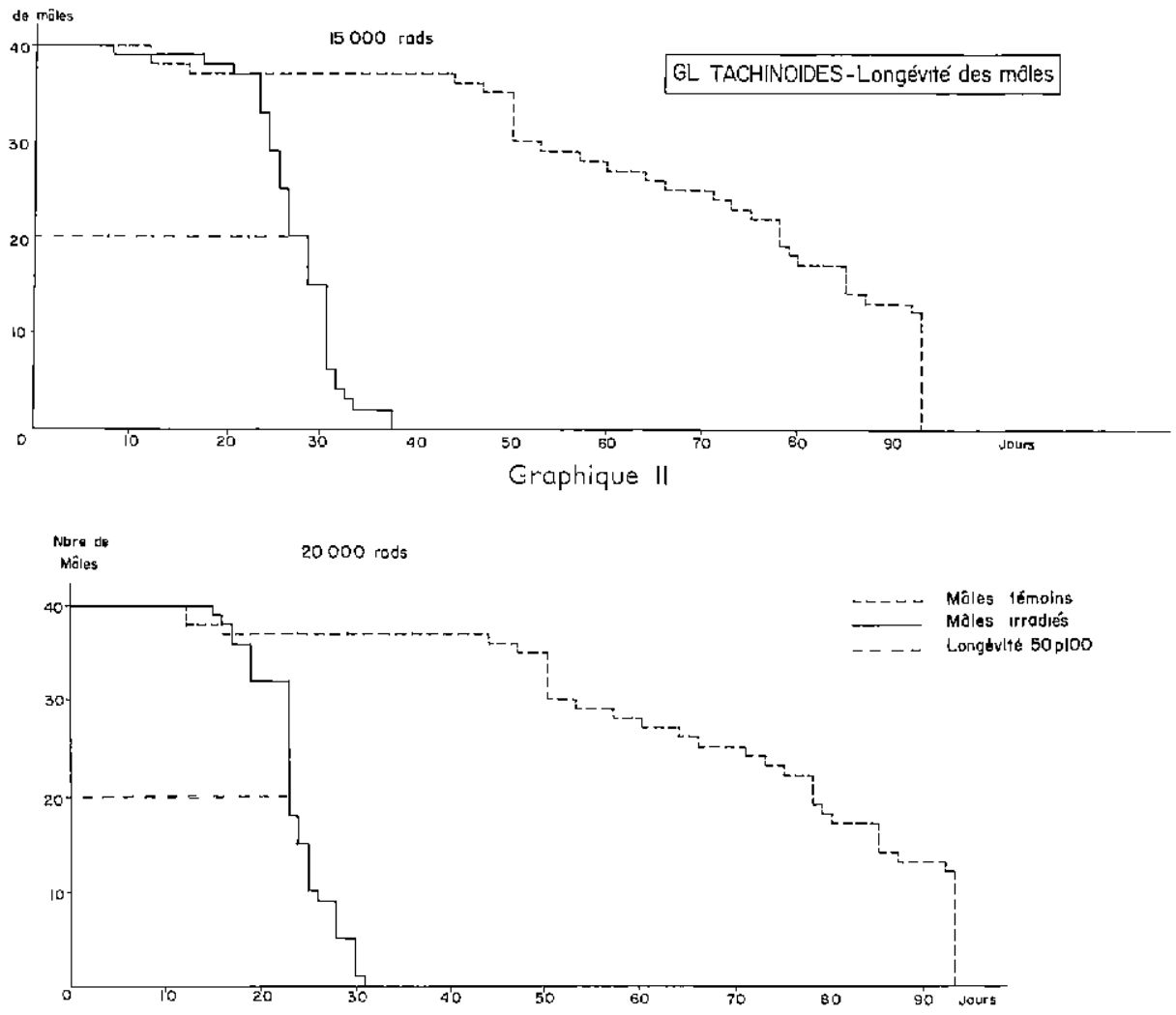

Mubre de
Mäles

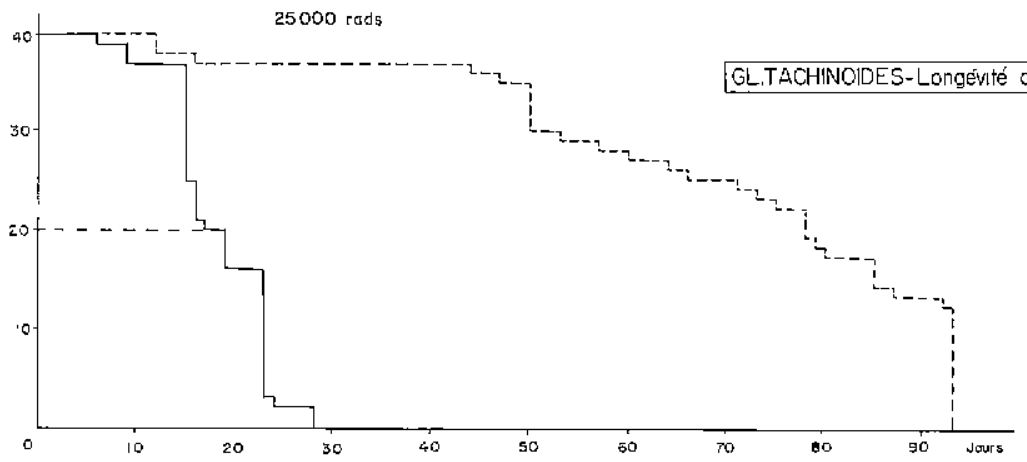

Graphique III 
Chez les mâles irradiés, la longévité décroît en fonction de la dose reçue. La longévité 50 p. 100 diminue rapidement entre 2.000 et 10.000 rads, puis plus lentement entre 10.000 et 25.000 rads. La longévité maximum est également inversement proportionnelle à la dose reçue (graphique 4). Par contre, quelle que soit la dose administrée, la mortalité est très faible au cours des dix premiers jours qui suivent l'irradiation, et n'est pas significativement différente de celle des mâles témoins (Tableau II).

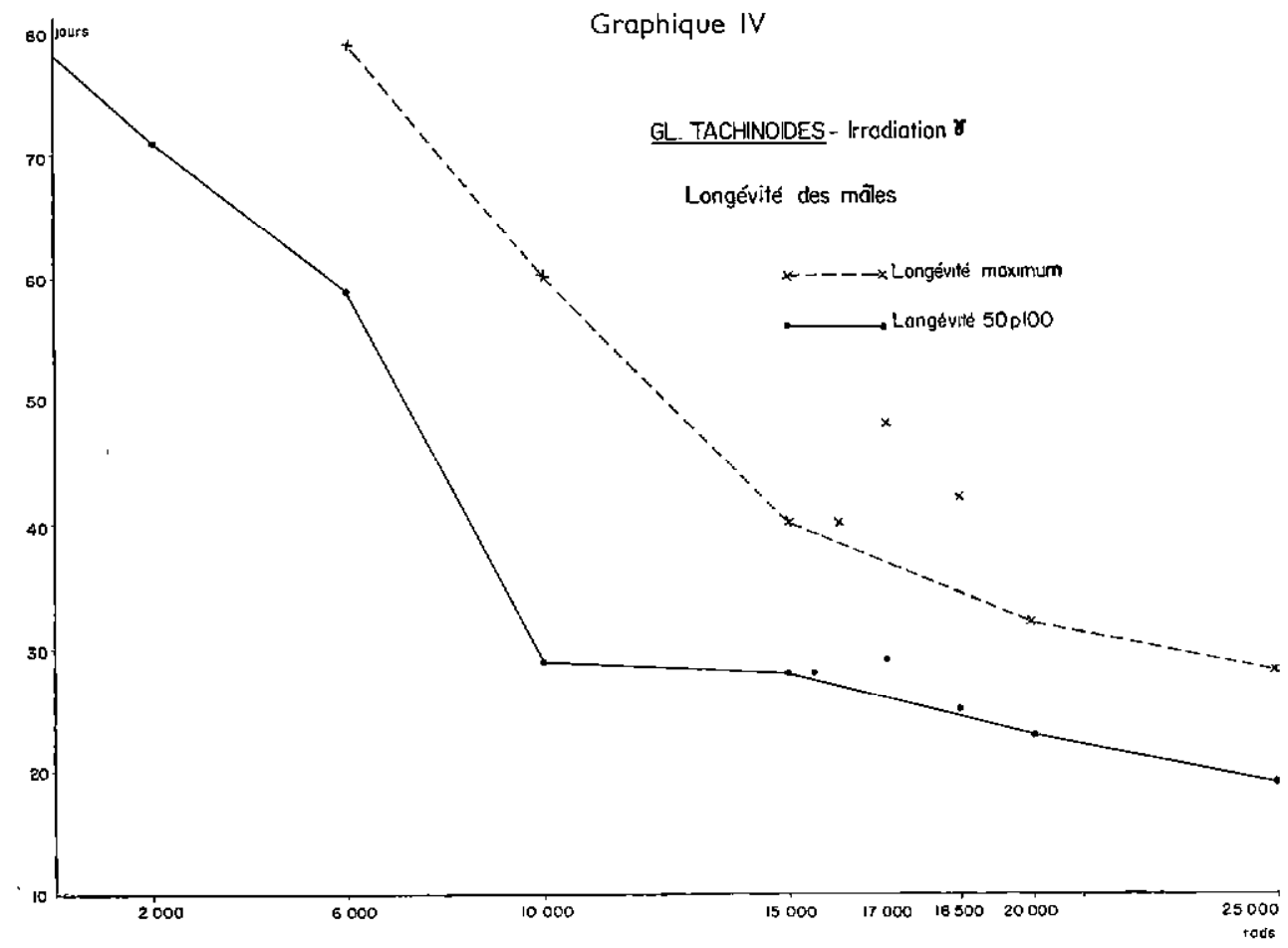

TABLEAU No II. - Longévité des mâles témoins et des mâles irradiés

\begin{tabular}{|c|c|c|c|c|c|}
\hline \multirow{2}{*}{$\begin{array}{l}\text { Do s e } s \\
\text { en rads }\end{array}$} & \multicolumn{2}{|c|}{$\begin{array}{l}\text { Survivants } 10 \text { fours } \\
\text { après } 1 \text { 'irradiation }\end{array}$} & \multirow{2}{*}{$\begin{array}{l}\text { Signification } \\
\qquad P=0,05\end{array}$} & \multirow{2}{*}{$\begin{array}{l}\text { Longévité } \\
50 \mathrm{p} \cdot 100\end{array}$} & \multirow{2}{*}{$\begin{array}{l}\text { Longévité } \\
\text { maximum }\end{array}$} \\
\hline & Nombre & Pourcentage & & & \\
\hline $\begin{array}{c}0 \\
\text { (Têmolns du 6.12) } \\
2.000 \\
6.000 \\
10.000 \\
15.000 \\
20.000 \\
25.000\end{array}$ & $\begin{array}{l}38 / 40 \\
37 / 40 \\
39 / 40 \\
38 / 40 \\
39 / 40 \\
40 / 40 \\
37 / 40\end{array}$ & $\begin{array}{r}95,00 \\
92,50 \\
97,50 \\
95,00 \\
97,50 \\
100,00 \\
92,50\end{array}$ & $\begin{array}{l}x^{2}=0,21 \\
\text { (d.d.1 }=5 \text { ) } \\
\text { N.S. }\end{array}$ & $\begin{array}{ll}78 & \text { jours } \\
71 & 11 \\
59 & 11 \\
29 & 1 \\
28 & 11 \\
23 & 11 \\
19 & 11\end{array}$ & $\begin{array}{l}\text { Supérieure à } 93 \mathrm{j} \text {. } \\
\text { Supérleure à } 93 \mathrm{j} \text {. } \\
79 \text { jours } \\
60 " \text { " } \\
40 " 1 \\
31 \quad " \\
28 \text { " }\end{array}$ \\
\hline $\begin{array}{c}0 \\
\text { (Têmodns du 5.3) } \\
15.500 \\
17.000 \\
18.500\end{array}$ & $\begin{array}{l}37 / 40 \\
34 / 40 \\
35 / 38 \\
36 / 40\end{array}$ & $\begin{array}{l}92,50 \\
85,00 \\
92,10 \\
90,00\end{array}$ & $\begin{array}{l}x^{2}=0,27 \\
(d, d, 1=2) \\
\text { N.s. }\end{array}$ & $\begin{array}{l}77 \text { jours } \\
28 \text { " } \\
29 \\
25\end{array}$ & $\begin{array}{l}\text { Supêrleure à } 93 \mathrm{j} \text {. } \\
\qquad \begin{array}{l}40 \text { fours } \\
47 \\
41\end{array}\end{array}$ \\
\hline
\end{tabular}




\section{2. - Stérilité des mâles.}

Le contrôle de la stérilité a été effectué par comparaison entre le nombre de pupes produites au bout de 60 jours par des femelles normales accouplées avec les mâles témoins ef le nombre de pupes produites dans le même temps par des femelles normales accouplées avec les mâles irradiés.

Chaque lot de mâles a été, en fonction du

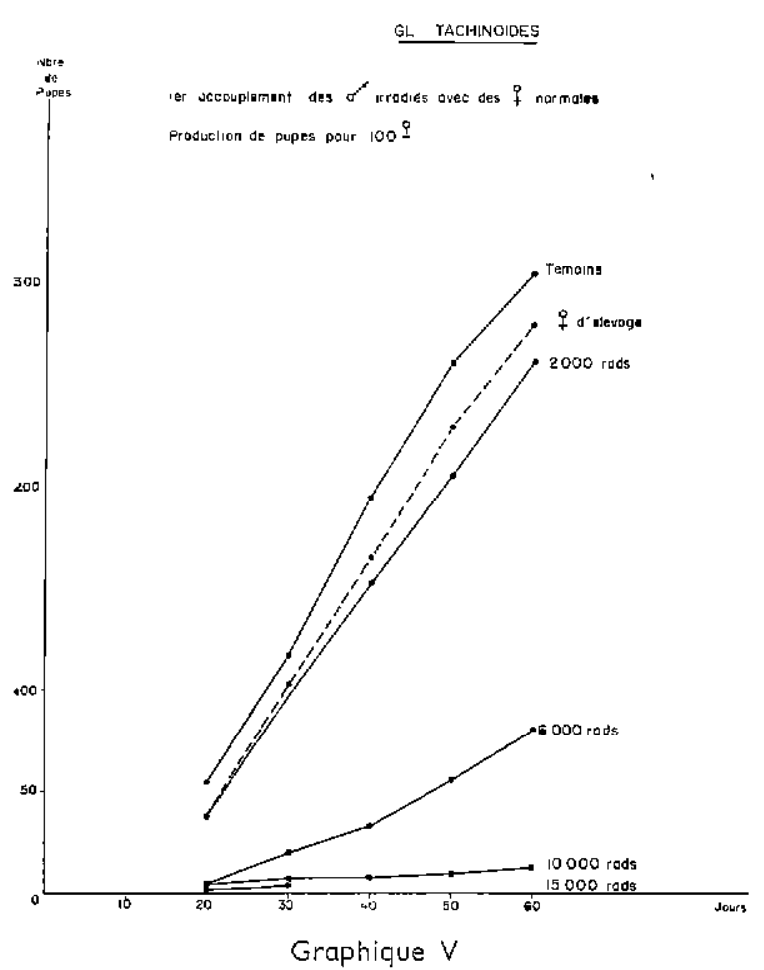

Les femelles accouplées avec les mâles témoins ont produit entre 308 et 335 pupes pour 100 femelles, en 60 jours. Les femelles d'élevage ont produit pendant les périodes correspondantes, entre 282 pupes pour 100 femelles accouplées ( 760 pupes pour 269 femelles) et 320 pupes pour 100 femelles accouplées (641 pupes pour 200 femeiles). Les femelles accouplées avec les mâles irradiés ont produit un nombre de pupes d'autant plus faible que la dose d'irradiation reçue par les mâles a été plus élevée. Au-delà de 15.500 rads, aucune production de pupes n'a été enregistrée.

Dans le graphique 7 nous avons figuré le nombre moyen de pupes par femelle reproduc- nombre de femelles disponibles, entre le $2^{-}$ et le $9 \mathrm{e}$ jour suivant l'irradiation, mis en présence d'un nombre égal de femelles normales âgées de 3 jours. La période de réunion des sexes a duré de 4 à 7 jours. Les mâles ont été alors séparés des femelles et réaccouplés avec de nouvelles femelles normales âgées de 3 jours.

Les productions de pupes en 60 jours, ramenées à 100 femelles accouplées, sont indiquées dans le Tableau iti et les grophiques 5 et 6 .

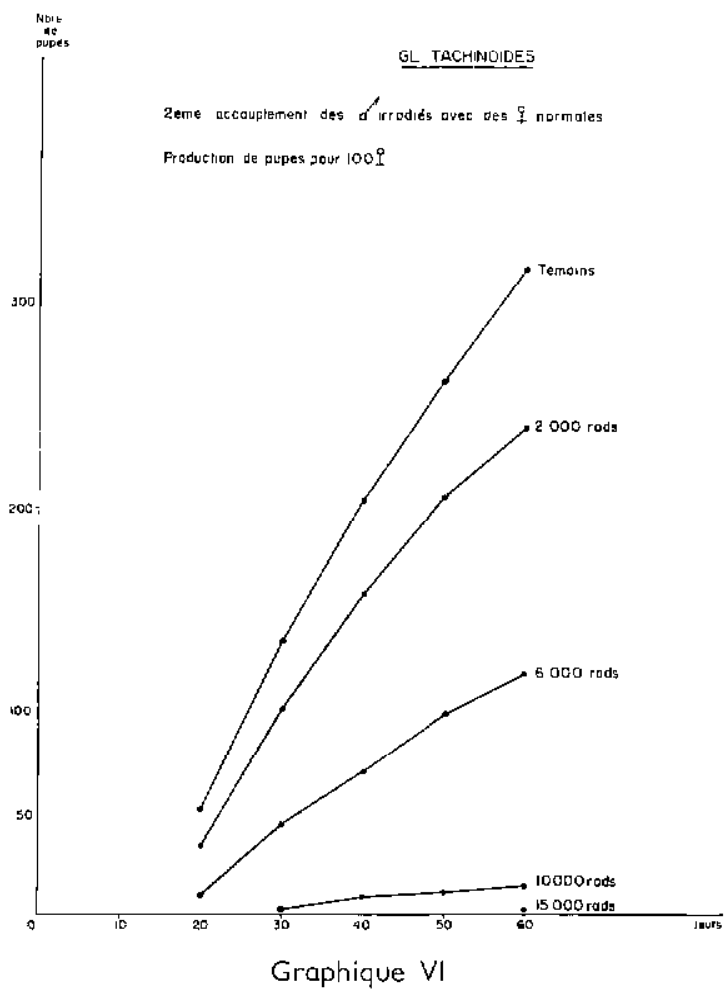

trice, en 60 jours. Ce chiffre a été obtenu en rapportant le nombre total de pupes produites, en 60 jours, au nombre moyen de femelles vivantes entre le jour 11 et le jour 60, diminué éventuellement, du nombre de femelles dont les spermathèques, à la dissection, ne contenaient aucun spermatozoïde (Tableau IV).

Afin de tenir compte des différences statistiques dans les productions de pupes de femelles normales accouplěes avec des mâles normaux, nous avons fait figurer le nombre de pupes par femelle le plus faible obtenu avec les femelles d'élevage (282 pupes pour 100 femelles accouplées ou 3,41 pupes par femelle reproductrice). 
TABLEAU $\mathrm{N}^{0}$ III

Femelles normales accouplées avec les mâles irradiês - Production de pupe日

\begin{tabular}{|c|c|c|c|c|c|c|c|c|c|c|c|c|c|c|c|c|c|c|}
\hline \multirow[t]{2}{*}{ Jours } & \multicolumn{3}{|c|}{$\begin{array}{c}\text { Têmoins } \\
\text { (let accouplement) } \\
\text { (1ot du } 6.12 .67)\end{array}$} & \multicolumn{3}{|c|}{$\begin{array}{c}2.000 \text { rads } \\
\text { (ler accouplement) }\end{array}$} & \multicolumn{3}{|c|}{$\begin{array}{l}6.000 \text { rads } \\
\text { (ler accouplement) }\end{array}$} & \multicolumn{3}{|c|}{$\begin{array}{l}10.000 \text { rads } \\
\text { (1er accouplement) }\end{array}$} & \multicolumn{3}{|c|}{$\begin{array}{l}15,000 \text { rads } \\
\text { (1er accouplement) }\end{array}$} & \multicolumn{3}{|c|}{$\begin{array}{c}15.500 \text { rads } \\
\text { (ler accoup lement) }\end{array}$} \\
\hline & 1 & 2 & 3 & I & 2 & 3 & 1 & 2 & 3 & 1 & 2 & 3 & 1 & 2 & 3 & 1 & 2 & 3 \\
\hline 0 & 38 & & & 37 & & & 38 & & & 39 & & & 39 & & & 36 & & \\
\hline $11-20$ & 33,6 & 21 & 55,26 & 37,0 & 14 & 37,80 & 36,0 & 2 & 5,21 & 35,3 & 2 & 5,06 & 38,0 & 1 & 2,53 & 34,0 & & \\
\hline $21-30$ & 32,1 & 24 & 118,35 & 34,6 & 23 & 99,82 & 36,0 & 6 & 20,93 & 32,5 & 1 & 7,55 & 38,0 & 1 & 5,12 & 34,0 & & \\
\hline $31-40$ & 30.7 & 30 & 197,27 & 31,6 & 20 & 153,79 & 35,9 & $s$ & 34,06 & 30,9 & 0 & 7,55 & 37,5 & & & 33,9 & & \\
\hline $41-50$ & 24,9 & 25 & 263,05 & 29,3 & 20 & 207,79 & 35,0 & 7 & 52,48 & 26,6 & 1 & 10,07 & 35,1 & & & 33,0 & 1 & 2,75 \\
\hline $51-60$ & 19,8 & 17 & 307,75 & 25,1 & 21 & 264,49 & 34,3 & 11 & 81,36 & 25,0 & 1 & 12,63 & 33,0 & & & 32,7 & 1 & 5,47 \\
\hline \multirow[t]{2}{*}{ Jours } & \multicolumn{3}{|c|}{$\begin{array}{c}\text { Témoins } \\
\text { (2è accouplement) } \\
\text { (lot du } 6.12 .67)\end{array}$} & \multicolumn{3}{|c|}{$\begin{array}{c}2.000 \text { rads } \\
\text { (2è accouplement) }\end{array}$} & \multicolumn{3}{|c|}{$\begin{array}{c}6.000 \mathrm{rads} \\
(2 \mathrm{e} \text { accoup lement })\end{array}$} & \multicolumn{3}{|c|}{$\begin{array}{c}10.000 \text { rads } \\
(2 \grave{e} \text { accouplement } t)\end{array}$} & \multicolumn{3}{|c|}{$\begin{array}{c}15,000 \text { rads } \\
(2 \mathrm{e} \text { accoup I ement })\end{array}$} & \multicolumn{3}{|c|}{$\begin{array}{c}\text { Témoing } \\
\text { (ler accouplement) } \\
(1 \text { ot du } 5,3,68)\end{array}$} \\
\hline & 1 & 2 & 3 & 1 & 2 & 3 & 1 & 2 & 3 & 1 & 2 & 3 & 1 & 2 & 3 & 1 & 2 & 3 \\
\hline 0 & 37 & & & 35 & & & 38 & & & 32 & & & 37 & & & 40 & & \\
\hline $11-20$ & 35,0 & 19 & 51,26 & 34,3 & 12 & 34,20 & 37,6 & 4 & 10,48 & 30,7 & & & 34,1 & & & 38,8 & 20 & 49.95 \\
\hline $21-30$ & 33,2 & 31 & 134,96 & 31,5 & 24 & 102,69 & 36,0 & 13 & 44,67 & 28,5 & 1 & 3,11 & 31,5 & & & 37,5 & 27 & 117,45 \\
\hline $31-40$ & 31,2 & 26 & 205,19 & 28,3 & 20 & 159.77 & 33,1 & 10 & 70,97 & 27,7 & 2 & 9,34 & 30,4 & & & 36,0 & 29 & 189,90 \\
\hline $41-50$ & 27,8 & 22 & 264,61 & 26,4 & 17 & 208,26 & 30,3 & 11 & 99,91 & 26,3 & 1 & 12,46 & 26,5 & & & 36,0 & 29 & 262,35 \\
\hline $51-60$ & 24.5 & 20 & 318,63 & 24,4 & 12 & 242,48 & 27,2 & 8 & 120,95 & 21,8 & 1 & 15,52 & 23,3 & 1 & 2,64 & 35,1 & 29 & 334,83 \\
\hline
\end{tabular}

$I$ = Nombre moyen de femelles/jour par décade , 2 n Nombre de pupes produites par décade, 3 = Nombre de pupes pour 100 femelles (Totaux cumulës). 


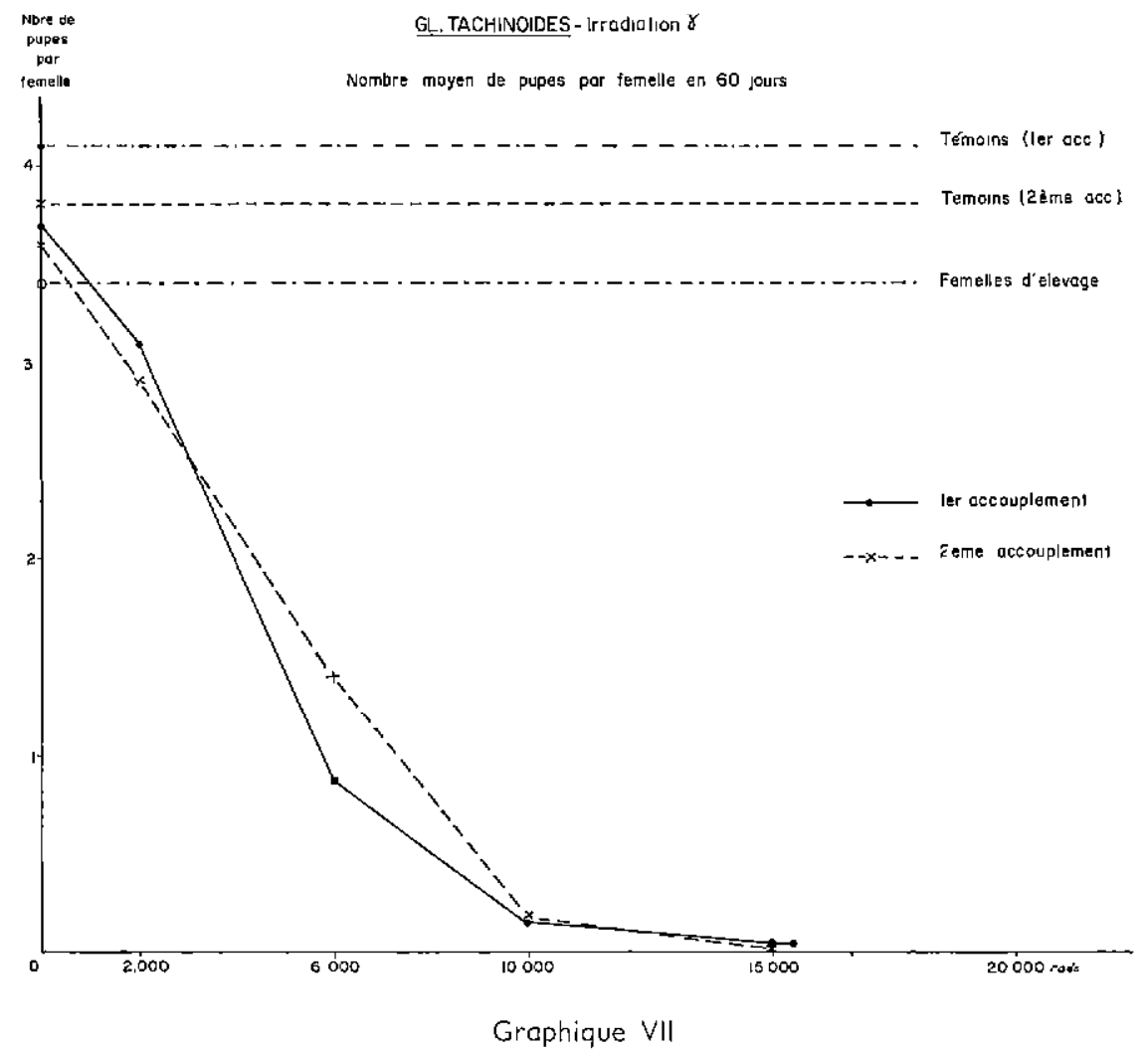

TABLEAU $\mathrm{N}^{0}$ IV

Nombre moyen de pupes par femelle reproductrice.

\begin{tabular}{|c|c|c|c|c|c|c|}
\hline \multirow{2}{*}{$\begin{array}{l}\text { Mâles } \\
\text { Doses } \\
\text { d'irradiation }\end{array}$} & \multicolumn{2}{|c|}{$\begin{array}{c}\text { Nombre moyen de } \\
\text { femelles reproductrices } \\
\text { (jour Il à jour } 60)\end{array}$} & \multicolumn{2}{|c|}{$\begin{array}{l}\text { Nombre de pupes } \\
\text { produites en } \\
60 \text { jours }\end{array}$} & \multicolumn{2}{|c|}{$\begin{array}{l}\text { Nombre de pupes } \\
\text { par femelle } \\
\text { reproductrice }\end{array}$} \\
\hline & $\begin{array}{c}\text { ler } \\
\text { accouplement }\end{array}$ & $\begin{array}{c}2 \grave{e} \\
\text { accouplement }\end{array}$ & $\begin{array}{c}\text { ler } \\
\text { accouplement }\end{array}$ & $\begin{array}{c}2 \overline{\mathrm{e}} \\
\text { accouplement }\end{array}$ & $\begin{array}{c}\text { ler } \\
\text { accouplement }\end{array}$ & $\begin{array}{c}\text { 2è } \\
\text { accouplement }\end{array}$ \\
\hline $\begin{array}{c}0 \\
\text { (Témoins du } 6.12 \text { ) }\end{array}$ & 28,22 & 30,34 & 117 & 118 & 4,14 & 3,88 \\
\hline $\begin{array}{c}0 \\
\text { (Témoins du 5, 3) }\end{array}$ & 36,68 & - & 134 & - & 3,65 & - \\
\hline (mâles d'êlevage) & 222,32 & - & 760 & - & 3,41 & - \\
\hline $2.000 \mathrm{rads}$ & 31,52 & 28,98 & 98 & 85 & $.3,10$ & 2,93 \\
\hline $6.000 \quad 11$ & 35,50 & 32,84 & 31 & 46 & 0,87 & 1,40 \\
\hline 10.000 & 30,06 & 27,00 & 5 & 5 & 0,16 & 0,18 \\
\hline 15.000 & 36,32 & 29,16 & 2 & 1 & 0,05 & 0,03 \\
\hline 15.500 & 33,52 & - & 2 & - & 0,08 & - \\
\hline 17.000 & 34,64 & - & D & - & 0,00 & - \\
\hline 18.500 & 35,94 & - & 0 & - & 0,00 & - \\
\hline 20.000 & 33,08 & 32,64 & 0 & 0 & 0,00 & 0,00 \\
\hline 25.000 & 31,66 & 19,48 & 0 & 0 & 0,00 & 0,00 \\
\hline
\end{tabular}


On constate ainsi :

a) que le nombre moyen de pupes par femelle reproductrice diminue lorsque la dose d'irradiation administrée aux mâles augmente ef est nul au-delà de 15.500 rads,

b) qu'il n'y a pas de différence significative entre le nombre de pupes produites par les femelles accouplées avec les mâles irradiés à 2.000 rads, et le nombre de pupes produites par les femelles accouplées avec les mâles témoins, mais cette différence est très hautement significative dès 6.000 rads $\left(x^{2}=130,56-d\right.$. d. I. $=1$. $P=0,001)$,

c) qu'il n'y a pas de différence significative entre les nombres de pupes produites après les deux accouplements successifs des mâles irradiés, y compris ceux ayant été irradiés à 6.000 rads $\left(\chi^{2}=3,67-\right.$ d. d. I. $\left.=1-P=0,05\right)$.

\section{3. - Capacité d'insémination des mâles.}

Les proportions obtenues, après dissection des femelles et examen au microscope des spermathèques, entre le nombre de femelles ayant leurs spermathèques parfaitement pleines, le nombre de femelles ayant leurs spermathèques partiellement remplies et le nombre de femelles ayant des spermathèques vides, permettent d'apprécier la capacité d'insémination des mâles.

Toutes les femelles accouplées avec les mâles témoins et les mâles irradiés ont été sacrifiées après le $60 \mathrm{e}$ jour ef disséquées. L'examen des spermathèques a permis de dresser les graphiques 8,9 et 10 ou sont figurées les proportions de spermathèques pleines (première colonne), de spermathèques partiellement pleines (deuxième colonne) et de spermathèques vides (troisième colonne) pour chacune des doses d'irradıation administrées aux mâles. Nous avons représenté, en tralts discontinus, les proportions correspondantes obtenues après dissection des femelles accouplées avec les mâles témoins.

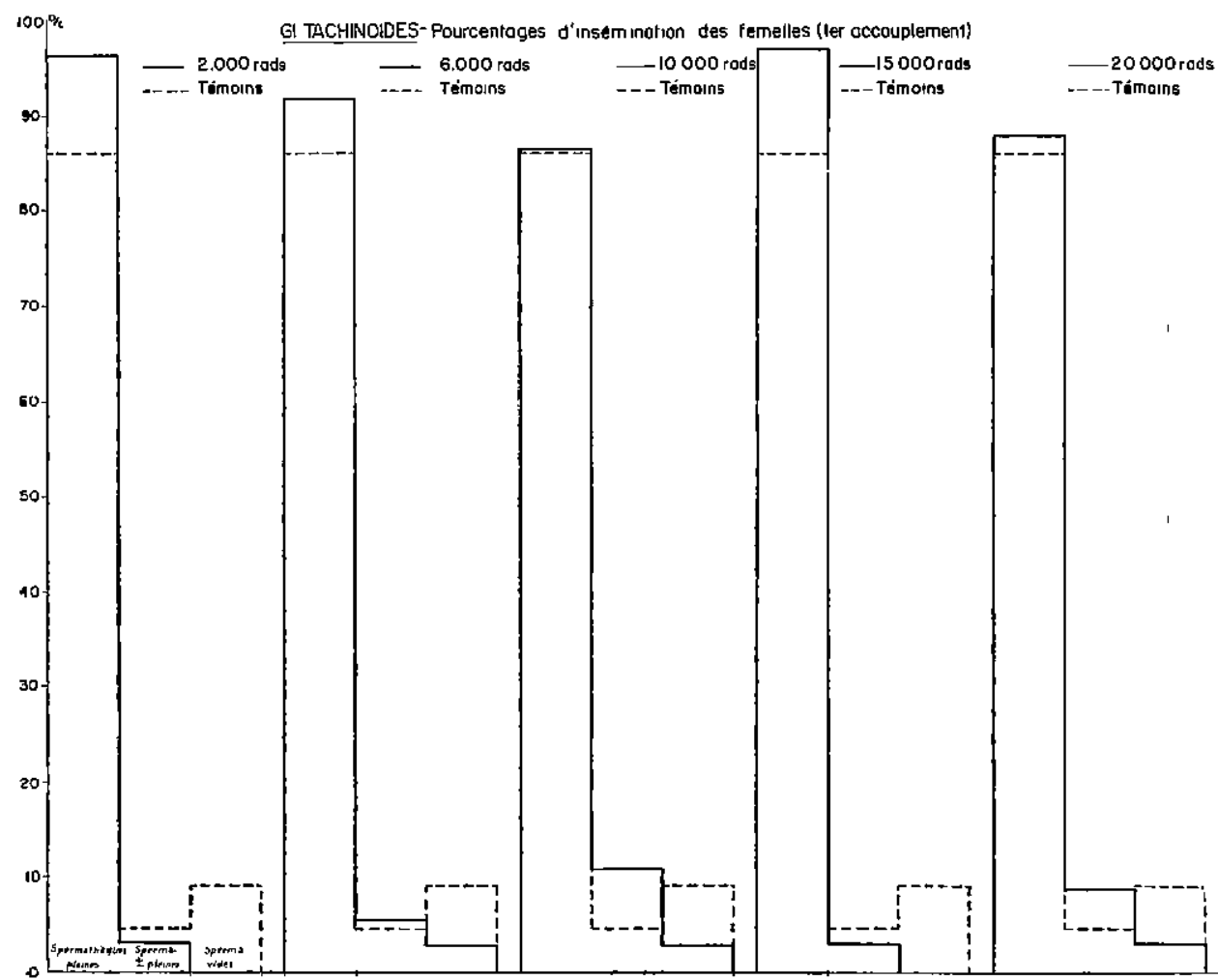

Grophique VIII 

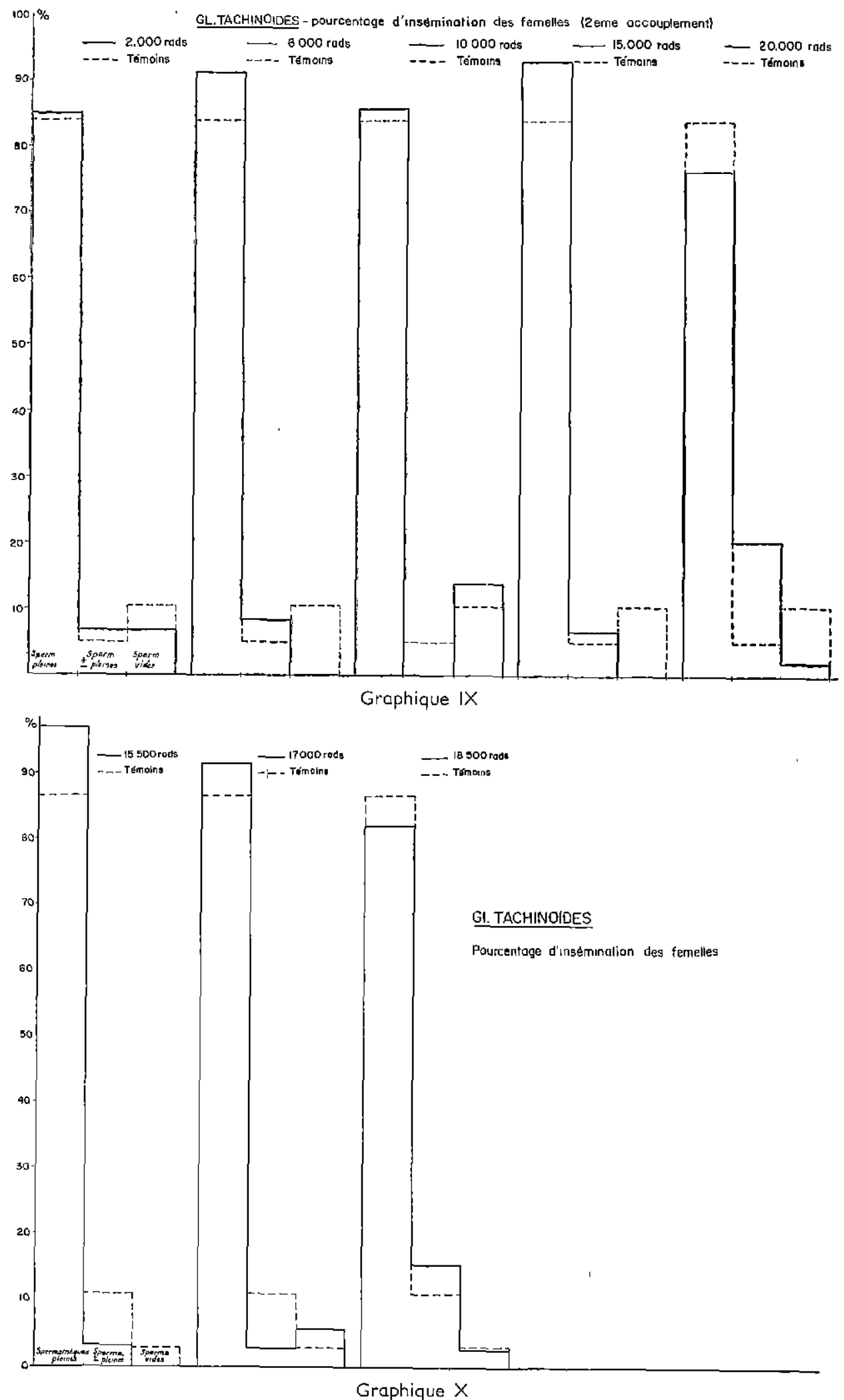
On constate que :

a) les mâles irradiés ont généralement une capacité d'insémination supérieure aux mâles témoins, même lors du $2^{\mathrm{e}}$ accouplement,

b) la capacité d'insémination est indépendante de la dose d'irradiation.

\section{4. - Mélange de mâles normaux et de mâles irradiés.}

Des lots de mâles irradiés à 15.500 rads, 17.000 rads et 18.500 rads ont été mélangés avec des mâles normaux dans les proportions suivantes :

a) 16 mâles irradiés ef 4 mâles normaux,

b) 12 males irradiés ef 8 mâles normaux,

c) 8 mâles irradiés et 12 mâles normaux,

d) 4 mâles irradiés ef 16 mâles normaux.

On a ainsi constitué 12 groupes comprenant chacun 20 mâles (quatre groupes de mélange pour chacune des trois doses précédentes). Chacun de ces groupes a été mis en présence de 20 femelles normales, vierges, aggées de 3 jours.

Les nombres moyens de pupes par femelle reproductrice, au bout de 60 jours, ont été les suivants :

\section{A. - Mâles irradiés d 15.500 rads :}

groupe $a=0,36$ pupe par femelle ( 6 pupes en 60 jours),

groupe $b=0,78$ pupe par femelle (15 pupes en 60 jours),

groupe $c=1,61$ pupes par femelle (33 pupes en 60 jours),

groupe $d=2,60$ pupes par femelle (46 pupes en 60 jours).

\section{B. - Mâles irrodiés à 17.000 rads :}

groupe $a=0.73$ pupe par femelle (14 pupes en 60 jours),

groupe $b=0,55$ pupe par femelle $(10$ pupes en 60 jours),

= groupe $c=1,24$ pupes par femelle (24 pupes en 60 jours),

groupe $d=2,17$ pupes par femelle ( 39 pupes en 60 jours).

\section{C. - Mâles irrodiés d 18.500 rads :}

groupe $a=0,82$ pupe par femelle (14. pupes en 60 jours),

groupe $b=1,00$ pupe par femelle (16 pupes en 60 jours),

groupe $c=2,07$ pupes par femelle ( 39 pupes en 60 jours),

groupe $d=2,25$ pupes par femelle (27 pupes en 60 jours).

Il apparaît ainsi que :

a) le nombre de pupes produites par femelle diminue, d'une façon générale, lorsque la proportion de mâles irradiés s'accrôt,

b) dans tous les cas et en particulier pour les groupes d ( 80 p. 100 de mâles normaux), le nombre de pupes produites par femelle reproductrice (2,34 en moyenne) a été inférieur au nombre de pupes produites par les femelles témoıns (3,65 pupes).

Dans les conditions de l'expérience, les mâles irradiés ont donc été pleinement compétitifs, et ont concurrencé de façon significative les mâles normaux.

\section{5. - Contrôle de la stérilité des femelles nor males accouplées avec des mâles irradiés.}

Les lots de mâles irradiés à 15.500 rads, 17.000 rads et 18.500 rads, accouplés une premıère fois avec des femelles normales, ont été réaccouplés une deuxième fols avec des femelles normales, vierges, âgées de 3 jours.

Aussitôt la séparation faite, ces femelles onł été mises en présence de mâles normaux âgés de 7 jours. La période de réunion des couples a duré 5 jours.

Les productions de pupes par femelle reproductrice, au bout de 60 jours, ont été respectivement de 0,34 pupe, 0,38 pupe et 0,46 pupe (Témoins $=3,65$ pupes).

Après dissection des femelles, examen de l'utérus et d'après les intervalles de temps séparant les pontes, il a été possible de déterminer dans chaque cas le nombre de femelles pondeuses, et par conséquent fertilisées par les mâles normaux (Tableau $V$ ). 
TABLEAU $\mathbb{N}^{\circ} \mathrm{V}$

\begin{tabular}{|c|c|c|c|c|c|c|}
\hline \multicolumn{2}{|c|}{ Mâles irradiés } & \multirow{2}{*}{$\begin{array}{r}\text { Nombre de } \\
\text { femeiles } \\
\text { normales }\end{array}$} & \multirow{2}{*}{$\begin{array}{l}\text { Nombre de } \\
\text { mâles } \\
\text { normaux }\end{array}$} & \multirow{2}{*}{$\begin{array}{l}\text { Nombre de } \\
\text { pupes en } \\
60 \text { jours }\end{array}$} & \multirow{2}{*}{$\begin{array}{l}\text { Pupe par } \\
\text { feme } 11 \text { e en } \\
60 \text { jours }\end{array}$} & \multirow{2}{*}{$\begin{array}{l}\text { Nombre de } \\
\text { femelles } \\
\text { ayant produit } \\
\text { des pupes }\end{array}$} \\
\hline Nombre & $\begin{array}{c}\text { Doses en } \\
\text { rads }\end{array}$ & & & & & \\
\hline 35 & 15.500 & 32 & 32 & 11 & 0,34 & 3 \\
\hline 35 & 17.000 & 34 & 37 & 13 & 0,38 & 3 \\
\hline 37 & 18.500 & 35 & 36 & 16 & 0,46 & 4 \\
\hline
\end{tabular}

Les mâles normaux ont fécondé entre 9 et 11 p. 100 des femelies précédemment accouplées avec des mâles irradiés. II est probable que ces femelles n'avaient en fait pas été inséminées lors du premier accouplement avec les mâles irradiés. Il est significatif à cet égard que le plus grand nombre de femelles pondeuses appartienne à la catégorle des femelles accouplées la première fois avec les mâles irradiés à 18.500 rads.

\section{6. - Action résiduelle des doses substérilisantes.}

Les pourcentages d'éclosion des pupes produites par les femelles accouplées avec les mâles témoins du 6 décembre et les femelles accouplées avec les mâles irradiés à $2.000,6.000$ et 10.000 rads sont respectivement de 92,18 p. 100 , 92,38 p. $100,83,77$ p. 100 et 83,33 p. 100.

II n'y a pas de différence significative entre les taux d'éclosion des pupes produltes par les femelles accouplées avec les mâles témoins et les taux d'éclosion des pupes produites, respectivement, par les femelies accouplées avec les mâles irradiés à 2.000 rads, 6.000 rads et $10.000 \operatorname{rads}\left(\chi^{2}=1,2-d . d .1 .=3-P=0,05\right)$.

Les adultes issus de ces pupes étaient apparemment normaux. II n'a pas été possible d'étudier la longévité et le pouvoir reproducteur de ces adultes. Cette étude fera l'objet de recherches ultérieures.

\section{IV. - CONCLUSIONS}

L'irradiation gamma de mâles adultes de Glossina tachinoides West., âgés de 24 h à 9 jours, à des doses comprises entre 2.000 rads et 25.000 rads, diminue à la fois la longévité et la fertilıté des mâles irradiés, proportionnellement d̀ la dose reçue.

Quelle que soit la dose administrée, la mortalité, dans les dix premiers jours qui suivent l'irradiation, reste cependant faible et n'est pas sıgnificatıvement différente de celle des mâles témoins.

La longévité 50 p. 100 des mâles irradiés oscille autour de 28 jours entre 15.000 ef 17.000 rads, mais n'atteint pas 20 jours à 25.000 rads, alors qu'elle dépasse 75 jours chez les mâles témoins.

Les productions de pupes des femelles normales accouplées avec les mâles irradiés diminuent lorsque les doses d'irradıation administrées aux mâles augmentent, et à partir de 6.000 rads sont significativement différentes du nombre de pupes produites par les femelles accouplées avec les mâles témoins. Les femelles accouplées avec des mâles irradiés à des doses supérieures à 15.500 rads ne produisent aucune pupe.

II n'y a pas de différence significative entre le nombre de pupes produites lors de deux accauplements successifs avec les mâles irradiés.

Jusqu'à 18.500 rads la capacité d'insémination des mâles irradiés est supérieure à celle des mâles témoins, et est indépendante de la dose d'irradiation.

Dans des mélanges en différentes proportions de mâles normaux et de mâles irradiés, ces dcrniers se sont montrés pleinement compétitifs.

Lorsque des femelies normales accouplées une première fols avec des mâles irradiés à $15.500,17.000$ et 18.500 rads ont été réaccouplées avec des mâles normaux, les productions de pupes ont été très faibles et proviennent vraisemblablement de femelles qui n'avaient pas été inséminées lors du premier accouplement. 
Dans les conditions de l'expérience, les doses d'irradiation optimales se situent entre 15.500 et 17.000 rads.

A ces doses, la fertilité des mâles irradiés est nulle, mass leur compétitivité et leur capacité d'insémination sont conservées et leur longévité reste satisfaisante.
Ces doses sont inférieures à celles qui sont nécessaires pour obtenir une stérilité totale des mâles de Gl. morsitans.

\section{Laboratoire d'Entornologie}

Institut d'Elevage ef de Médecine Vétérınoire des Pays Tropicaux.

\section{SUMMARY \\ Sterilisation of Glossina lachinoides West. \\ males through irradiation with gamma rays.}

The consequence of gamma Irradiation of adult Glossina tochinoides West. males have been studied for the practical use of the sterile-male release technique for control of this species.

The optimal doses of irradiation are between 15.500 and 17.000 rads. With these doses, males are completely sterilized, but their sexual behaviour is unchanged and their longivity still satısfactory.

These doses are lower than those necessary to obtain a complete sterility of Glossina morsitons morsitans Newst. male.

\section{RESUMEN}

\section{Esterillzación de los machos de Glossina fachinoides West. mediante una irradiación con rayos gamma}

Se estudiaron las consecuencias de la ırradıación gamma de machos aduitos de Glossina tachinoides West., con el objeto de una aplicación próctica del método de lucha por la suelta de machos estérıles.

Las dosis de irradiación optimas se siłùan entre 15.500 y 17.000 rads. Con estas dosis, la esterilidad de los machos es completa, pero su vigor sexual no está disminuido y su longevidad queda satisfactoria.

Dichas dosis san inferiores a las que se necesitan para obtener una esterilıdad completa en los machos de Glossino morsitans morsifons Newst.

\section{BIBLIOGRAPHIE}

DAME (D. A.), DEAN (G. J.W.) et FORD (J.).-Investigations of the sterile male technique with Glossina morsitans. Proc. 10th Mtg. international Scientific Committee for Trypanosomiasis Research (Kampala, Uganda), 1964, 93-96.

ITARD (J.). - Premiers résultats d'un essai d'irradiation gamma sur des pupes et des mâles adultes de Gl. morsitans morsitans. Groupe d'étude sur la lutte contre les Insectes nuisibles au bétail par la technique du lacher de mâles stériles ; Division conjointe
F.A.O./I.A.E.A., Vienne (Autriche), 1967, 23-27 janvier 1967.

ITARD (J.), MAILLOT (L.), BRUNET (J.) et GIRET (M.). - Observations sur un élevage de Glossina tachinoides West, après adoption du lapin comme animal-hôte. Rev. Elev. Méd. véf. Pays trop., 1968, 21 (3).

PHELPS (R. J.). - The sterile male technique in relation to tse-tse control. Rhodesion Scientific Association, 1967, 52 (1), 29-32.

POTTS (W. H.). - Sterilisation of tse-tse flies (Glossina) by gamma radiation. Ann. Trop. Méd. Parasit. 1958, 52, 484-499. 